\title{
ELECTROMAGNETIC AND RADIATIVE CASSON FLUID FLOW OVER A PERMEABLE VERTICAL RIGA-PLATE
}

\author{
Parasuraman Loganathan, Krishnamurthy Deepa \\ Department of Mathematics, College of Engineering, Guindy, Anna University, Chennai, Tamil Nadu, India \\ e-mail: logu@annauniv.edu; deepakkrish1@gmail.com
}

\begin{abstract}
The convective Casson fluid flow past a Riga plate is investigated theoretically. The flow properties are elucidated through the analytical solutions of transport equations which are acquired by adopting the Laplace transform technique. This study mainly focuses on nonlinear flow characteristics under radiation, permeability and the wall parallel Lorentz force. The positive modified Hartmann number refers to the aiding flow which enhances the flow speed. The comparison between Newtonian and non-Newtonian fluid flow subject to the wall parallel Lorentz force has also been done graphically. Further, the results of this Casson fluid flow model correlated with the vertical flow under buoyancy effect.
\end{abstract}

Keywords: Casson fluid, Riga plate, wall parallel Lorentz force, electromagnetism

\section{Introduction}

Yield-pseudo plastic materials generate a nonlinear flow curve together with particular yield stress. This type of material displays an infinite rate of shear with low apparent viscosity; much less (approaches to zero) shear rate is noticed at infinite apparent viscosity. The Casson fluid model associated with this viscoplastic behavior is defined as a shear thinning non-Newtonian fluid. In many industrial processes, this is used to expound the shear stress and shear rate characteristics of the fluid flow pertaining to yoghurt, tomato puree, food stuffs and biological materials (Chhabra and Richardson, 1999). Due to the high consistency of non-Newtonian fluids, they are processed under laminar flow conditions. A pseudo-plastic fluid exhibits a decrease in viscosity with the increasing rate of shear. Paints, printing inks and disperse systems are the most renowned examples of this kind of flow behavior. However, when the rate of shear is less, the poor flow speed is encountered. Because of this fact, pseudo plasticity is somehow unenviable (Cross, 1965). The yield stress is the more favourable quantity for the shear thinning fluids. That being so, the viscoplastic fluids proffered the higher deformation rate.

The Casson fluid model was invented by Casson who has formulated the constitutive equation which is reasonably successful in describing the flow curves for suspension of pigments in Silicon suspension and printing inks. On account of its real time usages, much attention has been given to this model recently. Exact solution of the Casson fluid flow past an oscillating vertical plate with Newtonian heating was obtained by Hussanan et al. (2014). It was found that higher Prandtl number regulated the flow separation. Numerical simulation of the Casson flow through a stenosed artery was done by Venkatesan et al. (2014).

At high temperature circumstances, thermal radiation has significant role in changing mechanical properties of complex non-Newtonian fluids. Radiation is also a regulating element of thermal energy in industrial processes. The Casson fluid flow along with suction or blowing and thermal radiation at a stretching surface exhibits high temperature for an elevation in the radiation parameter; likewise the stagnation flow displays the enhanced heat transfer rate (Pramanik, 2014; Ramesh et al., 2016). 
Boundary layer control is essentially important, when the fluid flow acts differently than its usual characteristics. Suction, blowing and motion of the wall are active control methods which have the substantial impact on the flow pattern. These classical methods delay the boundary layer separation from the surface. This irregular flow behaviour can be controlled by electromagnetism, which offers powerful practical flow control due to high electrical conductivity of the material (Dubovikova et al., 2014). The external magnetic field already has high influence on the high electrical conductivity fluids in MHD fluid flow control. In the case of weakly conducting fluids, very less amount of current produced by the external magnetic field. Consequently, an external electric field has to be applied to attain convincing boundary layer control (Magyari and Pantokratoras, 2011).

A charged particle in a crossed electric and magnetic field experiences the flow by controlling the boundary layer growth. Gailitis and Lielausis from physics institute at Riga designed an electromagnetic control system which is a span wise alternate arrangement of electrodes and permanent magnets. This actuator is termed as the Riga-plate. The mixed convection study on the Riga-plate was performed by Magyari and Pantokratoras (2011) under the Lorentz force in the direction of the free stream velocity and opposite to it. The boundary layer equations were solved numerically by the finite difference method. It was concluded that the aiding flow range shoot up the velocity and the opposing flow range exhibits the velocity drop. Pantokratoras and Magyari (2009) focused on the effects of the wall parallel Lorentz force on a natural convective EMHD flow. On the other hand, Ganesh Kumar et al. (2017) studied the mass transfer characteristics of three dimensional flows.

Many recent studies on the Riga-plate contributed in the field of nanoscience. Specifically, the behavior of squeezed nanofluid flow over a Riga-plate subject to melting heat transfer, viscous dissipation and heat generation/absorption manifest the stronger momentum boundary layer for uplifted modified Hartmann number; whereas the squeezed flow with convective boundary conditions encounter a drop in velocity distribution (Hayat et al., 2017a,b). Most of the investigations centralized their priority to numerical simulation, owing to the complexity in deriving the exact solution. Recently, authors (Ahmad et al., 2016, 2017; Abbas et al., 2016; Hayat et $a l ., 2016)$ who constructed the physical model on Riga-plate were focused only on numerical solutions.

Many mathematical models addressed some critical issues in handling errors while the numerical evaluation has been carried out. In this context, obtaining the exact solution to the physical problem is the distinctive feature of this study. Hari and Patel (2016) and Kumar et al. (2016) acquired the exact solution of convective fluid flow over an oscillating and moving porous plate, respectively. No evaluation for the exact solution has been made for the Casson fluid flow over a permeable Riga-plate in the vertical sense.

The unique aspect of the present investigation is the analysis of some physical insight into the Casson flow mechanism over a moving vertical Riga-plate. The time dependent rheological characteristics measured under thermal radiation and permeability of the porous medium. A typical comparison between Newtonian and non-Newtonian fluid flow past the Riga-plate and validation of evaluated results with the already published work has also been performed.

\section{Mathematical analysis}

Consider the laminar buoyancy driven Casson fluid flow over a permeable impulsively started semi-infinite vertical Riga-plate. Assume that the plate is at rest initially. Hence, it is evident that the fluid temperature and concentration remain the conditions at the surroundings. The plate suddenly starts to accelerate from repose vertically upwards to gain a constant speed $u_{0}$ at $t^{\prime}>0$. This impulsive motion results in the velocity, temperature and concentration. At 
this time level, the temperature of the plate raises to $T_{w}^{\prime}\left(>T_{\infty}^{\prime}\right)$ and the concentration at the vicinity of the plate increases to $C_{w}^{\prime}\left(>C_{\infty}^{\prime}\right)$. Eventually, the fluid encounters the heat and mass transfer near the wall. For the case that the flow is far away from the plate, the temperature and concentration decline to free stream conditions and the velocity gradually drops to zero. The fluid motion is taken along the $x$ direction vertically, and the $y$ direction is normal to the plate. The velocities $u$ and $v$ are described along the vertical and horizontal directions respectively. The schematic representation is shown in Fig. 1. The effect of viscous dissipation is negligible in the energy balance equation. The stream-wise direction is insignificant in radiative flux and scattering is neglected.

(a)

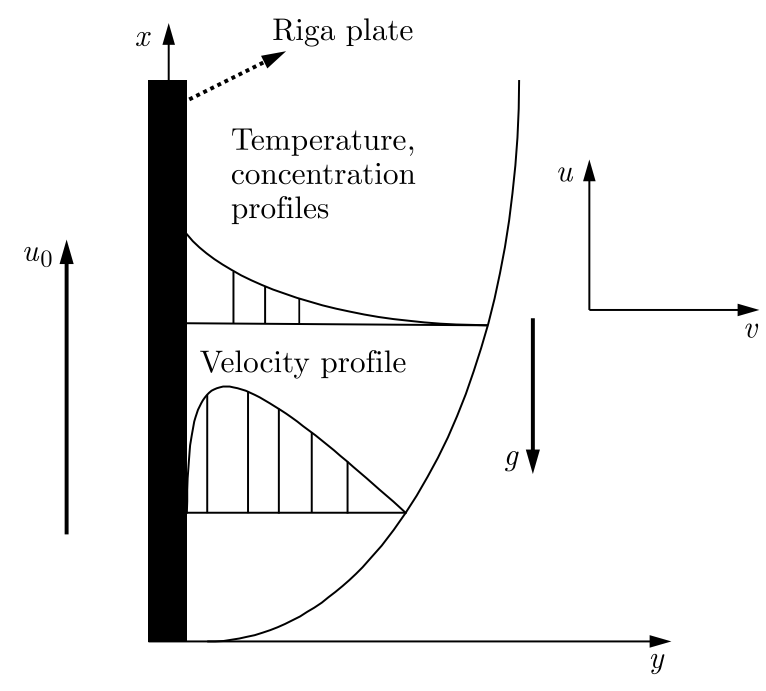

(b)

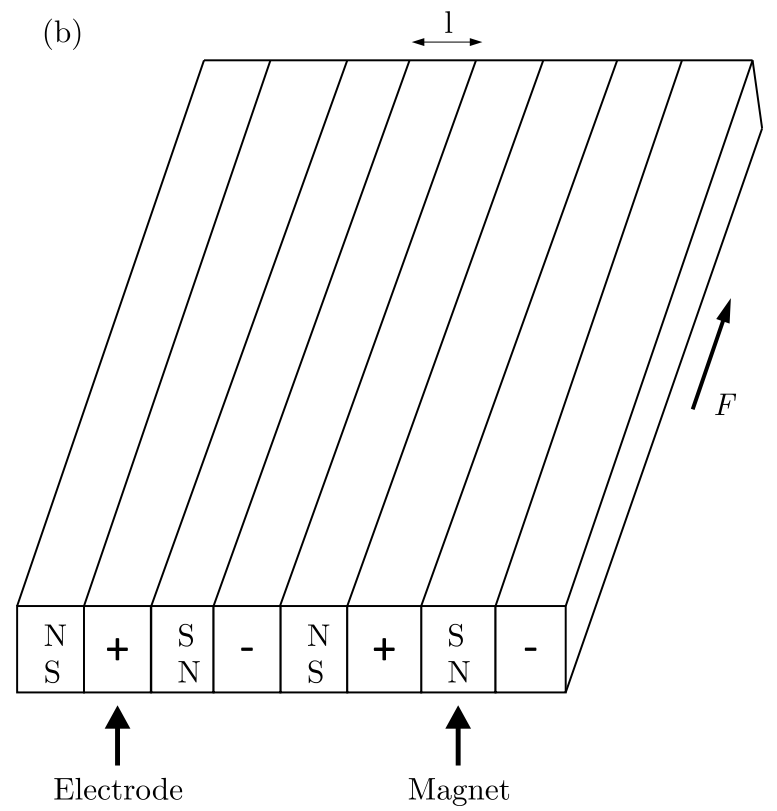

Fig. 1. (a) Schematic representation of the flow model, (b) arrangement of electrodes and magnets in the Riga-plate

The expression for the flow configuration of the Casson fluid is defined by a constitutive relation (Makanda et al., 2015; Arthur et al., 2015; Raju et al., 2016; Ullah et al., 2016)

$$
\tau_{i j}=\left\{\begin{array}{cc}
2\left(\mu_{B}+\frac{P_{y}}{\sqrt{2 \pi}}\right) e_{i j} & \pi^{\prime}>\pi_{c} \\
2\left(\mu_{B}+\frac{P_{y}}{\sqrt{2 \pi_{c}}}\right) e_{i j} & \pi^{\prime} \leqslant \pi_{c}
\end{array}\right.
$$

Here, $\pi^{\prime}=e_{i j} e_{i j}$ and $e_{i j}$ is the $(i, j)$-th component of the shear rate. $\pi_{c}$ is the critical value of $\pi^{\prime}$ concerned with the model. $\mu_{B}$ is the plastic dynamic viscosity and $P_{y}$ is the yield stress of the fluid.

The governing boundary layer equations of the mathematical model that encompasses the above assumptions, the constitutive equation and Boussinesq approximation can be expressed as

$$
\begin{aligned}
& \frac{\partial u}{\partial t^{\prime}}=\nu\left(1+\frac{1}{\beta}\right) \frac{\partial^{2} u}{\partial y^{2}}+g \beta^{*}\left(T^{\prime}-T_{\infty}^{\prime}\right)+g \beta^{\prime}\left(C^{\prime}-C_{\infty}^{\prime}\right)+\frac{\pi J_{0} M_{0} e^{-\frac{\pi}{l} y}}{8 \rho}-\frac{u \nu}{k} \\
& \frac{\partial T^{\prime}}{\partial t^{\prime}}=\alpha \frac{\partial^{2} T^{\prime}}{\partial y^{2}}-\frac{1}{\rho C_{p}} \frac{\partial q_{r}}{\partial y} \quad \frac{\partial C^{\prime}}{\partial t^{\prime}}=D \frac{\partial^{2} C^{\prime}}{\partial y^{2}}
\end{aligned}
$$


Suitable initial and boundary conditions of the problem are

$$
\begin{aligned}
& \left\{t^{\prime} \leqslant 0, u=0, T^{\prime}=T_{\infty}^{\prime}, C^{\prime}=C_{\infty}^{\prime}\right\} \quad \text { for all } y \\
& \left\{t^{\prime}>0, u=u_{0}, T^{\prime}=T_{w}^{\prime}, C^{\prime}=C_{w}^{\prime}\right\} \quad \text { for } y=0 \\
& \left\{u \rightarrow 0, T^{\prime} \rightarrow T_{\infty}^{\prime}, C^{\prime} \rightarrow C_{\infty}^{\prime}\right\} \quad \text { as } y \rightarrow \infty
\end{aligned}
$$

where $\beta=\mu_{B} \sqrt{2 \pi_{c}} / P_{y}$ is the Casson parameter, $\nu$ is the kinematic viscosity, $g$ is the gravitational acceleration, $\beta^{*}$ is the thermal expansion coefficient, $\beta^{\prime}$ is the volumetric coefficient of mass diffusion, $J_{0}$ is the current density, $M_{0}$ is the magnetization of magnets, $l$ is the width of the magnets and electrodes, $k$ is the permeability, $\alpha$ is the thermal diffusivity, $C_{p}$ is the specific heat, $q_{r}$ is the radiative flux, $D$ is the mass diffusion rate, $\rho$ is the density, $u, T^{\prime}, C^{\prime}$ and $t^{\prime}$ are the dimensional velocity, temperature, concentration and time, respectively.

In the energy balance equation, $\partial q_{r} / \partial y$ denotes the change in the radiative flux of the non-scattering medium in the direction normal to the plate. According to (England and Emery, 1969; Brewster, 1992; Pattnaik et al., 2017), this can be expressed as

$$
\frac{\partial q_{r}}{\partial y}=-4 a^{*} \sigma\left(T_{\infty}^{\prime 4}-T^{\prime 4}\right)
$$

where $a^{*}$ is the absorption coefficient, $\sigma$ is the Stefan Boltzmann constant.

The temperature difference in the flow is insubstantial such that $T^{\prime 4}$ can be written as a linear function of $T^{\prime}$ using the Taylor series approximation about $T_{\infty}^{\prime}$ (Ibrahim and Mankinde, 2011)

$$
T^{\prime 4} \simeq 4 T_{\infty}^{\prime 3} T^{\prime}-3 T_{\infty}^{\prime 4}
$$

Using (2.4) and (2.5) in $(2.2)_{2}$, the energy equation reduces to

$$
\frac{\partial T^{\prime}}{\partial t^{\prime}}=\alpha \frac{\partial^{2} T^{\prime}}{\partial y^{2}}+\frac{16 a^{*} \sigma T_{\infty}^{\prime 3}\left(T_{\infty}^{\prime}-T^{\prime}\right)}{\rho C_{p}}
$$

Non dimensional quantities employed on the coupled in partial differential equations $(2.2)_{1,3}$, (2.3), and (2.6) are taken as

$$
\begin{array}{lcc}
U=\frac{u}{u_{0}} \quad Y=\frac{y u_{0}}{\nu} \quad t=\frac{t^{\prime} u_{0}^{2}}{\nu} \quad T=\frac{T^{\prime}-T_{\infty}^{\prime}}{T_{w}^{\prime}-T_{\infty}^{\prime}} \\
C=\frac{C^{\prime}-C_{\infty}^{\prime}}{C_{w}^{\prime}-C_{\infty}^{\prime}} \quad G r=\frac{\nu g \beta^{*}\left(T_{w}^{\prime}-T_{\infty}^{\prime}\right)}{u_{0}^{3}} \quad \operatorname{Pr}=\frac{\nu}{\alpha} \\
N=\frac{16 a^{*} \sigma T_{\infty}^{\prime 3} \nu^{2}}{\kappa u_{0}^{2}} \quad G c=\frac{\nu g \beta^{\prime}\left(C_{w}^{\prime}-C_{\infty}^{\prime}\right)}{u_{0}^{3}} \quad S c=\frac{\nu}{D} \\
K=\frac{k u_{0}^{2}}{\nu^{2}} \quad P=\frac{\pi J_{0} M_{0} \nu}{8 \rho u_{0}^{3}} \quad S=\frac{\pi \nu}{l u_{0}}
\end{array}
$$

where $U, T, t$ and $Y$ are the dimensionless velocity, temperature, time and spatial coordinate along the transverse direction respectively. $G r, G c, P r, S c, K, P, N$ and $\kappa$ are the thermal Grashof number, mass Grashof number, Prandtl number, Schmidt number, permeability parameter, modified Hartmann number, radiation parameter and thermal conductivity, respectively.

Non-dimensional forms of $(2.2)_{1,3},(2.3)$, and (2.6) are given by

$$
\begin{aligned}
& \frac{\partial U}{\partial t}=\left(1+\frac{1}{\beta}\right) \frac{\partial^{2} U}{\partial Y^{2}}+G r T+G c C+P e^{-S Y}-\frac{U}{K} \\
& \frac{\partial T}{\partial t}=\frac{1}{P r} \frac{\partial^{2} T}{\partial Y^{2}}-\frac{N T}{P r} \quad \frac{\partial C}{\partial t}=\frac{1}{S c} \frac{\partial^{2} C}{\partial Y^{2}}
\end{aligned}
$$


Appropriate initial and boundary conditions are

$$
\begin{aligned}
& \{t \leqslant 0, U=0, T=0, C=0\} \quad \text { for all } Y \\
& \{t>0, U=1, T=1, C=1\} \quad \text { for } Y=0 \\
& \{U \rightarrow 0, T \rightarrow 0, C \rightarrow 0\} \quad \text { as } Y \rightarrow \infty
\end{aligned}
$$

Equations (2.8) together with the initial and boundary conditions (2.9) are transformed by employing the Laplace transform, and the flow properties are retrieved in terms of $Y, t$ while using an inverse transform on the transformed equations. The expressions which demonstrate the flow behavior are obtained as follows:

- mass diffusion

$$
C(Y, t)=\operatorname{erfc}\left(\sqrt{\frac{S c}{t}} \frac{Y}{2}\right)
$$

— temperature distribution

$$
T(Y, t)=\frac{1}{2}\left[e^{-Y \sqrt{N}} \operatorname{erfc}\left(\sqrt{\frac{P r}{t}} \frac{Y}{2}-\sqrt{\frac{N t}{P r}}\right)+e^{Y \sqrt{N}} \operatorname{erfc}\left(\sqrt{\frac{P r}{t}} \frac{Y}{2}+\sqrt{\frac{N t}{P r}}\right)\right]
$$

— velocity distribution

$$
U(Y, t)=F_{1}(Y, t)+F_{2}(Y, t)+F_{3}(Y, t)+F_{4}(Y, t)
$$

where

$$
\begin{aligned}
& F_{1}(Y, t)=\frac{1}{2}\left[e^{-Y \sqrt{\frac{b_{1}}{a}}} \operatorname{erfc}\left(\frac{Y}{2 \sqrt{a t}}-\sqrt{b_{1} t}\right)+e^{Y \sqrt{\frac{b_{1}}{a}}} \operatorname{erfc}\left(\frac{Y}{2 \sqrt{a t}}+\sqrt{b_{1} t}\right)\right] \\
& F_{2}(Y, t)=\frac{G r}{a b_{2} b_{4}}\left\{\frac{1}{2}\left[e^{-Y \sqrt{\frac{b_{1}}{a}}} \operatorname{erfc}\left(\frac{Y}{2 \sqrt{a t}}-\sqrt{b_{1} t}\right)+e^{Y \sqrt{\frac{b_{1}}{a}}} \operatorname{erfc}\left(\frac{Y}{2 \sqrt{a t}}+\sqrt{b_{1} t}\right)\right]\right. \\
& -\frac{e^{-b_{4} t}}{2}\left[e^{-Y \sqrt{\frac{b_{1}-b_{4}}{a}}} \operatorname{erfc}\left(\frac{Y}{2 \sqrt{a t}}-\sqrt{\left(b_{1}-b_{4}\right) t}\right)+e^{Y \sqrt{\frac{b_{1}-b_{4}}{a}}} \operatorname{erfc}\left(\frac{Y}{2 \sqrt{a t}}+\sqrt{\left(b_{1}-b_{4}\right) t}\right)\right] \\
& -\frac{1}{2}\left[e^{-Y \sqrt{P r b_{5}}} \operatorname{erfc}\left(\frac{Y \sqrt{P r}}{2 \sqrt{t}}-\sqrt{b_{5} t}\right)+e^{Y \sqrt{P r b_{5}}} \operatorname{erfc}\left(\frac{Y \sqrt{P r}}{2 \sqrt{t}}+\sqrt{b_{5} t}\right)\right] \\
& +\frac{e^{-b_{4} t}}{2}\left[e^{-Y \sqrt{P r\left(b_{5}-b_{4}\right)}} \operatorname{erfc}\left(\frac{Y \sqrt{P r}}{2 \sqrt{t}}-\sqrt{\left(b_{5}-b_{4}\right) t}\right)\right.
\end{aligned}
$$

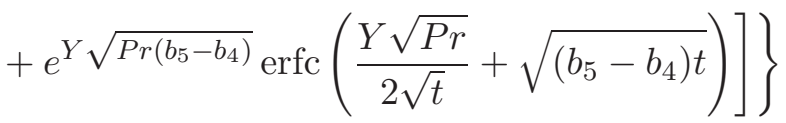

$$
\begin{aligned}
& F_{3}(Y, t)=\frac{G c}{a b_{6} b_{7}}\left\{\frac { e ^ { b _ { 7 } t } } { 2 } \left[e^{-Y \sqrt{\frac{b_{1}+b_{7}}{a}}} \operatorname{erfc}\left(\frac{Y}{2 \sqrt{a t}}-\sqrt{\left(b_{1}+b_{7}\right) t}\right)\right.\right. \\
& \left.+e^{Y \sqrt{\frac{b_{1}+b_{7}}{a}}} \operatorname{erfc}\left(\frac{Y}{2 \sqrt{a t}}+\sqrt{\left(b_{1}+b_{7}\right) t}\right)\right]-\frac{1}{2}\left[e^{-Y \sqrt{\frac{b_{1}}{a}}} \operatorname{erfc}\left(\frac{Y}{2 \sqrt{a t}}-\sqrt{b_{1} t}\right)\right. \\
& \left.+e^{Y \sqrt{\frac{b_{1}}{a}}} \operatorname{erfc}\left(\frac{Y}{2 \sqrt{a t}}+\sqrt{b_{1} t}\right)\right]-\frac{e_{7}^{b} t}{2}\left[e^{-Y \sqrt{S c b_{7}}} \operatorname{erfc}\left(\frac{Y \sqrt{S c}}{2 \sqrt{t}}-\sqrt{b_{7} t}\right)\right. \\
& \left.\left.+e^{Y \sqrt{S c b_{7}}} \operatorname{erfc}\left(\frac{Y \sqrt{S c}}{2 \sqrt{t}}+\sqrt{b_{7} t}\right)\right]+\frac{e^{-Y \sqrt{S c}}+e^{Y \sqrt{S c}}}{2} \operatorname{erfc}\left(\frac{Y \sqrt{S c}}{2 \sqrt{t}}\right)\right\}
\end{aligned}
$$




$$
\begin{aligned}
F_{4}(Y, t) & =\frac{P}{b_{8}}\left\{\frac{1}{2}\left[e^{-Y \sqrt{\frac{b_{1}}{a}}} \operatorname{erfc}\left(\frac{Y}{2 \sqrt{a t}}-\sqrt{b_{1} t}\right)+e^{Y \sqrt{\frac{b_{1}}{a}}} \operatorname{erfc}\left(\frac{Y}{2 \sqrt{a t}}+\sqrt{b_{1} t}\right)\right]\right\} \\
- & \frac{P}{b_{8}}\left\{\frac { e ^ { b _ { 8 } t } } { 2 } \left[e^{-Y \sqrt{\frac{b_{1}+b_{8}}{a}}} \operatorname{erfc}\left(\frac{Y}{2 \sqrt{a t}}-\sqrt{\left(b_{1}+b_{8}\right) t}\right)\right.\right. \\
+ & \left.\left.e^{Y \sqrt{\frac{b_{1}+b_{8}}{a}}} \operatorname{erfc}\left(\frac{Y}{2 \sqrt{a t}}+\sqrt{\left(b_{1}+b_{8}\right) t}\right)\right]\right\}-e^{-S Y}\left(\frac{P}{b_{8}}-\frac{P e^{b_{8} t}}{b_{8}}\right)
\end{aligned}
$$

and

$$
\begin{aligned}
& a=1+\frac{1}{\beta} \quad b_{1}=\frac{1}{K} \\
& b_{2}=\operatorname{Pr}-\frac{1}{a} \\
& b_{3}=N-\frac{1}{K a} \\
& b_{4}=\frac{b_{3}}{b_{2}} \\
& b_{5}=\frac{N}{P r} \\
& b_{7}=\frac{1}{b_{6} K a} \\
& b_{8}=a s^{2}-b_{1}
\end{aligned}
$$

The expressions for Nusselt and Sherwood numbers are given by

$$
\begin{aligned}
& N u=-\left.\frac{\partial T}{\partial Y}\right|_{Y=0}=\sqrt{N}+\frac{\sqrt{P r} e^{-\frac{N t}{P r}}}{\sqrt{\pi t}}-\sqrt{N} \operatorname{erfc}\left(\sqrt{\frac{N t}{P r}}\right) \\
& S h=-\left(\frac{\partial C}{\partial Y}\right)_{Y=0}=\sqrt{\frac{S c}{\pi t}}
\end{aligned}
$$

The skin friction coefficient is given by

$$
C_{f}=-\left.\left(1+\frac{1}{\beta}\right) \frac{\partial U}{\partial Y}\right|_{Y=0}
$$

\section{Results and discussion}

Behaviour of the flow is illustrated through expressions (2.10)-(2.14) based on the values procured from MATLAB programming corresponding to variations in $Y$. Magyari and Pantokratoras (2011) explained the aiding and opposing flow at steady state on the Riga-plate. A similar analysis for a Casson fluid in an unsteady case subject to radiation and permeability leads to the non-linearity study. Figure 2 discusses the velocity for both aiding and opposing flows. The positive modified Hartmann number exhibits an increase in the speed of the flow. Physically, it refers to the fact that the wall parallel Lorentz force is acting along the uniform mainstream velocity. Whereas, the opposing flow decelerates the fluid flow due to the resistance caused by the increased viscosity. Typically, the reverting case occurs in opposite to the mainstream velocity. Based on the porous medium structure, the velocity distribution has been enhanced for higher values of the permeability parameter. This is because of the size of pores or the amount of pores present in the porous medium which are increased and which results in the bulk fluid motion, while improving permeability parameter. Radiation leads to a drop in the energy level which, in turn, displays the reduction in the velocity boundary layer. The resistance in the fluid flow is noticed, when the Prandtl number values are increased.

Figure 3a demonstrates the impact on the velocity distribution with fixed values of $\operatorname{Pr}=7.2$, $N=7, K=0.3$ and $P=2.5$. A rise in these values increases the velocity field. This is because of the temperature and concentration difference in the fluid respectively. The Casson parameter describes the relation between viscosity and yield stress of the fluid. By increasing the viscosity, an elevation in the Casson parameter is attained. Hence, higher values of the Casson parameter results in a thicker momentum boundary layer. For a large time level, the impulsive motion of the 


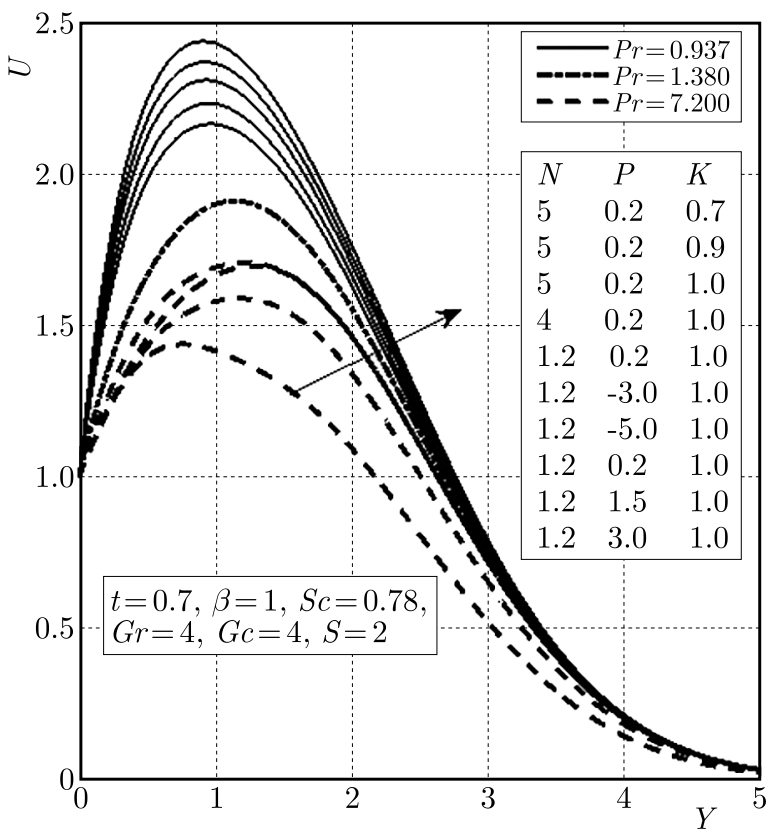

Fig. 2. Impact of $N, P, P r$ and $K$ on the velocity field

(a)

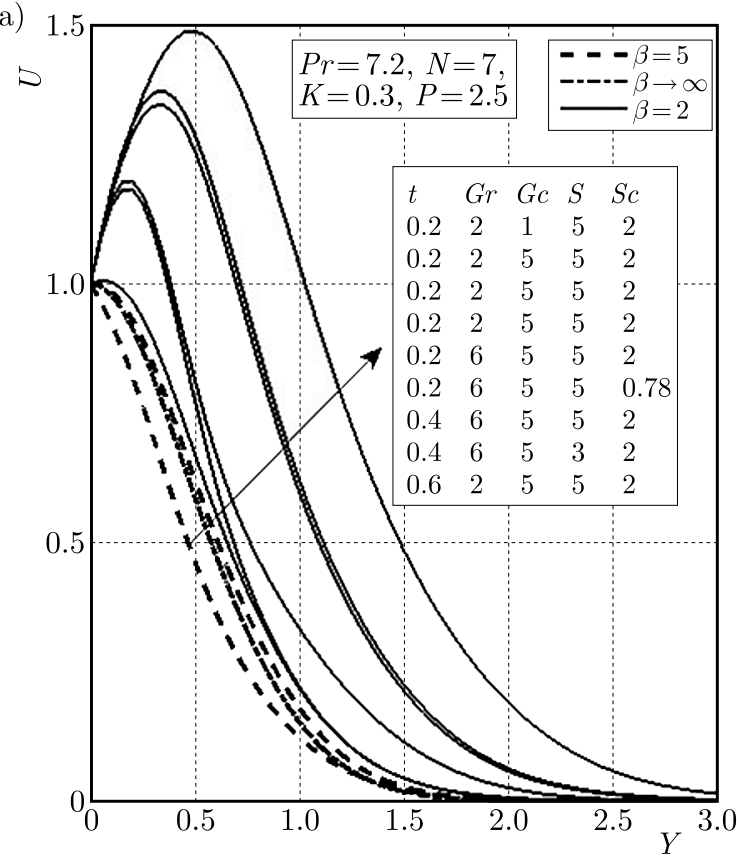

(b)

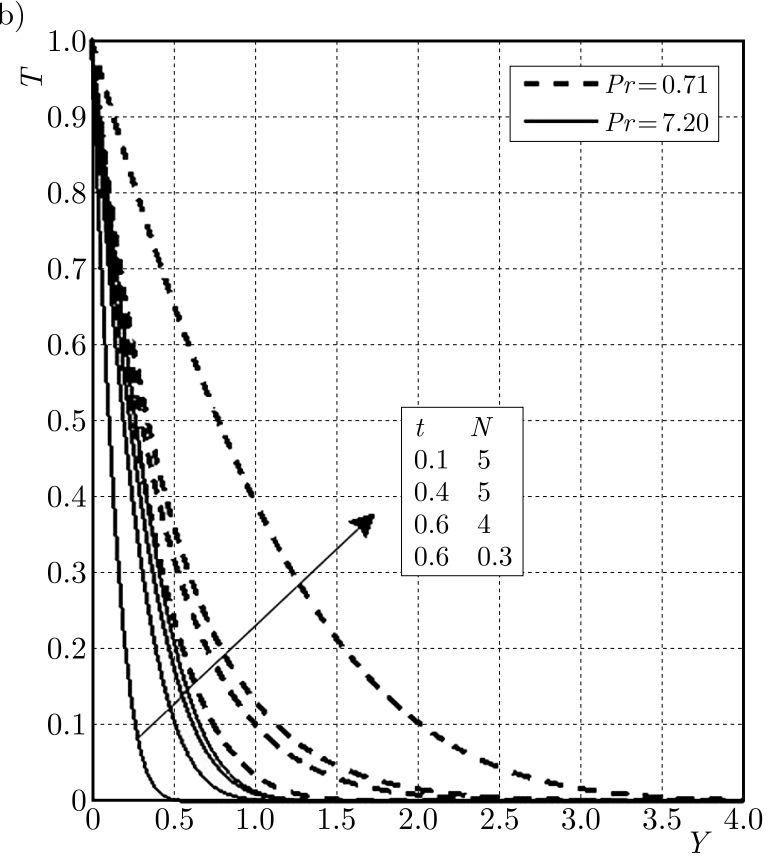

Fig. 3. (a) Influence of $t, G r, G c, S$ and $S c$ on velocity distribution. (b) Effects of $t, N$ and $\operatorname{Pr}$ on temperature distribution

plate shoots up the velocity. The dimensionless parameter $S$ defines the relation between viscosity and velocity of the plate with respect to width of the magnets and electrodes. The parameter $S$ and Schmidt number decrease the flow speed. Chhabra and Richardson (1999) reported that the physical properties of non-Newtonian fluids were normally temperature dependent. The temperature difference between the ambience and wall temperature dispersed the heat energy. Variation in the temperature is also generated by various pertinent parameters considered in this investigation. It is depicted in Fig. 3b. During energy transport by means of radiation, high energy electrons drop down to a lower energy level. This leads to the temperature drop. The 
uplifting Prandtl number results in reduction of thermal diffusivity. This affects the thermal boundary layer by lessening it. The temperature is raised for an elongated time.

Figure 4a elucidates the concentration distribution as a function of $Y$. By virtue of a lower molecular diffusion rate with higher values of the Schmidt number, a thinner mass transfer boundary layer is observed. For the prolonged period of time, the mass diffusion is enhanced. The rate of heat transfer has been analyzed for various values of the radiation parameter and Prandtl number in Fig. 4b. These two admissible parameters enhance the energy transport from high the temperature region to the low temperature region.

(a)

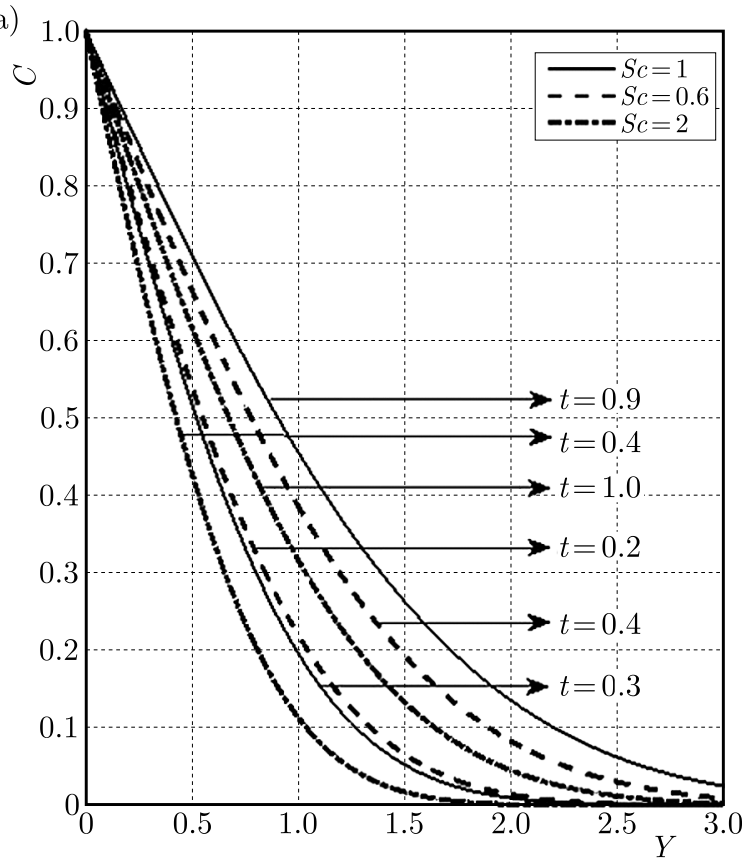

(b)

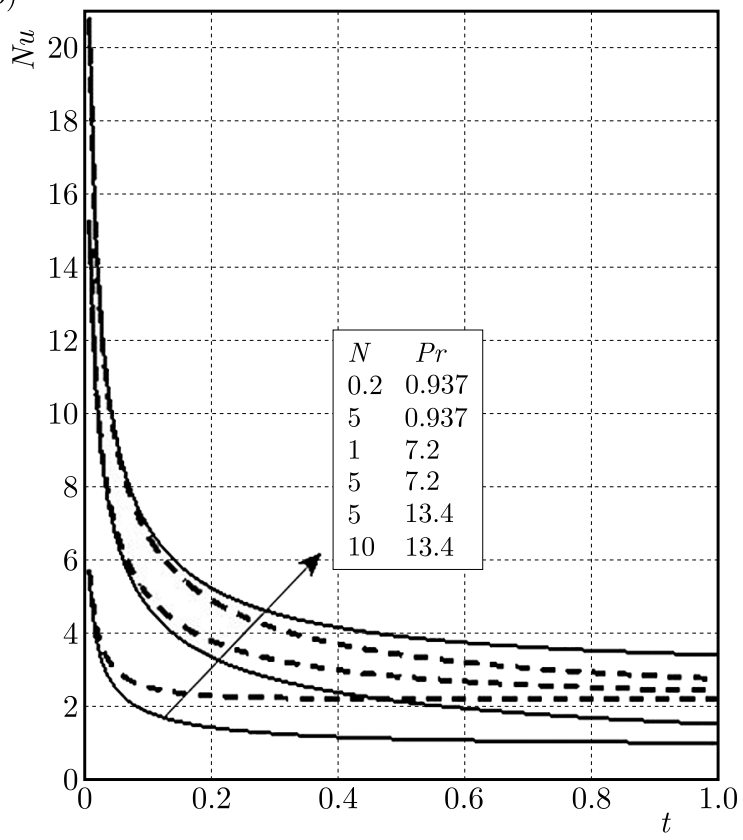

Fig. 4. (a) Mass diffusion for various time levels and Schmidt numbers. (b) Nusselt number variations with respect to $N$ and $P r$

Figure 5a presents the mass transfer rate for various values of the Schmidt number. The Schmidt number which is considered in this study represents various species at low concentration in air for approximately $25^{\circ} \mathrm{C}$ and $1 \mathrm{~atm}$, as specified by Gebhart and Pera (1971), Soundalgekar and Ganesan (1981), $S c=0.30(\mathrm{He}), 0.78\left(\mathrm{NH}_{3}\right), 1.24\left(\mathrm{So}_{2}\right), 2\left(\mathrm{C}_{7} \mathrm{H}_{16}\right)$. It is found that the mass transfer rate is escalated for heavier species. Irrespective of the fluid, hike in the values of $S c$ enhance the mass transfer rate correlated with variations in the concentration gradient. Figure $5 \mathrm{~b}$ reveals that the Newtonian and non-Newtonian fluids follow the same velocity trend while raising the modified Hartmann number. However, the flow speed is enhanced in the case of a non-Newtonian fluid by virtue of high yield stress exerted on the fluid flow.

Gebhart and Pera (1971) unveiled the most intriguing elements of the vertical flow exposed to natural convection. Results of this analysis obtained for water with $\operatorname{Pr}=S c=7, G r=G c$ are compatible with the current investigation for the values $\operatorname{Pr}=S c=7, G r=G c=2$, $P=N=0, K, \beta \rightarrow \infty$. It is illustrated in Fig. 6. As $\beta \rightarrow \infty$, the Casson fluid shows a Newtonian flow configuration.

Table 1 analyzes the viscous drag of the fluid flow owing to the high radiation parameter. The flow speed is escalated which results in the lower skin friction coefficient. Resistance to the flow occurs as the Casson parameter is increased. This phenomenon elevates the friction on the plate surface. An increase in the permeability parameter produces an increase in the skin friction coefficient as a consequence that the fluid can easily approach the plate surface through a large number of pores or bigger pores owing to the fact that the external electrical conductivity 
(a)

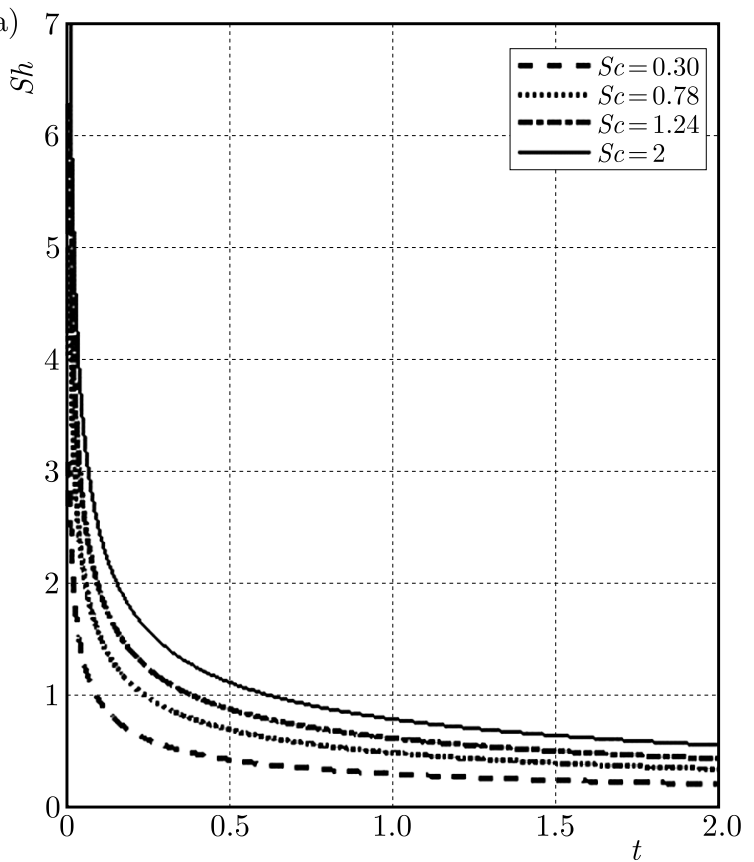

(b)

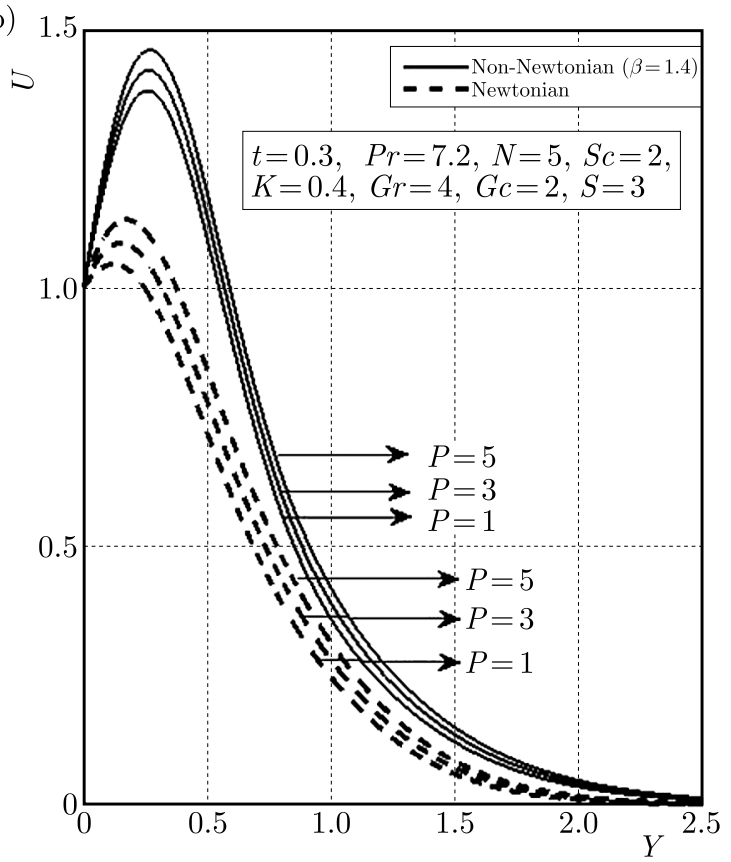

Fig. 5. (a) Influence of the Schmidt number on the mass transfer rate. (b) Comparison of Newtonian and non-Newtonian flow speed

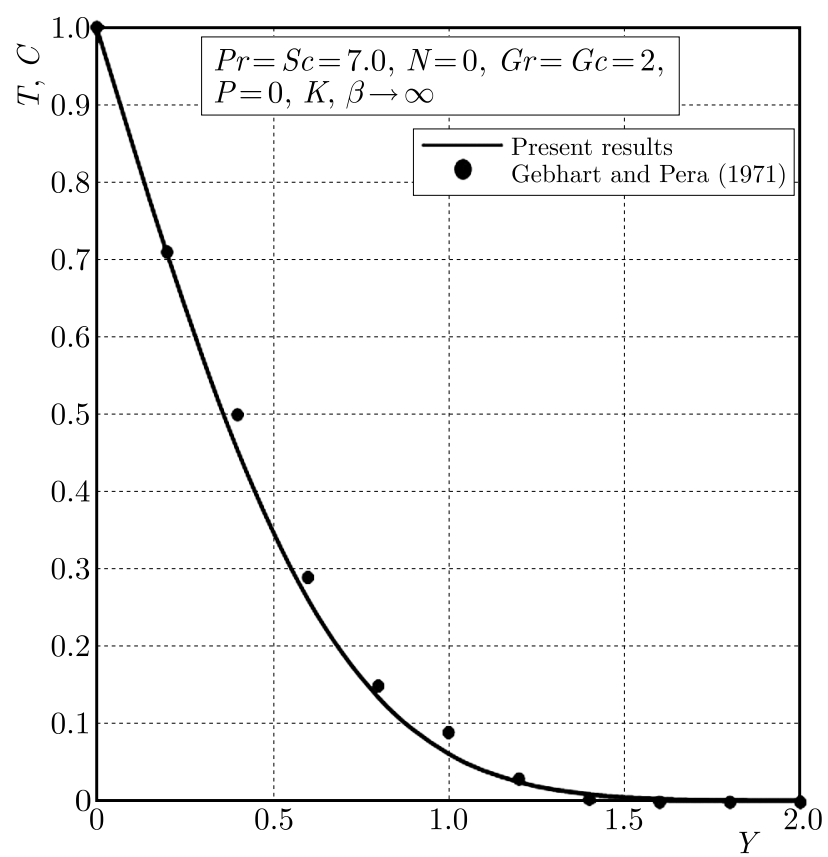

Fig. 6. Validation of the solution with Gebhart and Pera (1971)

lessens the viscous drag induced by the fluid flow. It is evident that the skin friction coefficient has mixed of positive and negative values. In particular, the positive values corresponding to $\beta=2.4$ with the fixed quantities $N=1, K=0.7$ and $P=1.5$ are related to the negative velocity gradient along the $Y$-direction. These two values betray the impact of $\beta$ on $C_{f}$. While improving $\beta$ from 0.3 to the reasonable amount, the viscosity is lifted up. For this reason, the fluid momentum decays monotonically with the negative velocity gradient. Thus for higher values of $\beta$, flow separation may be expected. The flow separation pertaining to the influence of the modified Hartmann number was discussed in detail by Magyari and Pantokratoras (2011). In order to introduce the distinctive discussion, the Casson parameter has been taken into account. 
Table 1. Skin friction coefficient for different values of $N, \beta, K$ and $P$ with $\operatorname{Pr}=7.2, S c=1.24$, $G r=4, G c=2, S=1.2$ and $t=0.8$

\begin{tabular}{|c|c|c|c|c|}
\hline$N$ & $\beta$ & $K$ & $P$ & $C_{f}$ \\
\hline \hline 0.1 & 0.3 & 0.7 & 1.5 & -1.50219 \\
\hline 1 & 0.3 & 0.7 & 1.5 & -1.36493 \\
\hline 3 & 0.3 & 0.7 & 1.5 & -0.96197 \\
\hline 1 & 0.3 & 0.7 & 1.5 & -1.36493 \\
\hline 1 & 2 & 0.7 & 1.5 & 1.16252 \\
\hline 1 & 4 & 0.7 & 1.5 & 1.63166 \\
\hline 10 & 1 & 0.3 & 1.5 & -2.44045 \\
\hline 10 & 1 & 0.5 & 1.5 & -1.17113 \\
\hline 10 & 1 & 0.7 & 1.5 & -1.00980 \\
\hline 5 & 4 & 0.7 & 1.5 & -2.07298 \\
\hline 5 & 4 & 0.7 & 2 & -2.73111 \\
\hline 5 & 4 & 0.7 & 3 & -4.04739 \\
\hline
\end{tabular}

\section{Conclusions}

From a theoretical standpoint, the buoyancy driven boundary layer flow of Casson fluid over a permeable Riga plate has been analyzed meticulously. The study emphasizes the rheological properties in a detailed manner. The summary of notable results is as follows:

- When the Lorentz force is pointing towards the mainstream velocity, the speed of the flow is enhanced. It is decaying for the reverting flow.

- The larger permeability enhances the velocity distribution and skin friction coefficient.

- Uplifting radiation parameter decreases the velocity and temperature, and elevates the viscous drag and Nusselt number.

- Fluid velocity is improved for escalated $G r, G c$ and $t$. On the other hand, the reverse trend has been noticed for $\beta, P r, S$ and $S c$.

- Temperature of the fluid is declined considerably for increased $\operatorname{Pr}$. However, it is elevated with time $t$.

- Improved Schmidt number shoots up the mass transfer rate although it diminishes the concentration boundary layer. Mass diffusion is progressively increased for elongated time.

- Increasing the Prandtl number substantially enhances the rate of heat transfer.

\section{Acknowledgements}

One of the authors, K. Deepa, immensely expresses the gratitude to Anna University, Chennai, for supporting this research work through "Anna Centenary Research Fellowship".

\section{References}

1. Abbas T., Ayub M., Bhatti M.M., Rashidi M.M., Ali M.E., 2016, Entropy generation on nanofluid flow through a horizontal Riga plate, Entropy, 223, DOI: 10.3390/e18060223

2. Ahmad A., Asghar S., Afzal S., 2016, Flow of nanofluid past a Riga plate, Journal of Magnetism and Magnetic Materials, 402, 44-48

3. Ahmad R., Mustafa M., Turkyilmazoglu M., 2017, Buoyancy effects on nanofluid flow past a convectively heated vertical Riga-plate: A numerical study, International Journal of Heat and Mass Transfer, 111, 827-835 
4. Arthur E.M., Seini I.Y., Bortteir L.B., 2015, Analysis of Casson fluid flow over a vertical porous surface with chemical reaction in the presence of magnetic field, Journal of Applied Mathematics and Physics, 3, 713-723

5. Brewster M.Q., 1992, Thermal Radiative Transfer and Properties, John Wiley \& Sons

6. Chhabra R.P., Richardson J.F., 1999, Non-Newtonian Flow in the Process Industries; Fundamentals and Engineering Applications, Butterworth-Heinemann Publishing

7. Cross M.M., 1965, Rheology of non-Newtonian fluids: A new flow equation for pseudoplastic systems, Journal of Colloid Science, 20, 417-437

8. Dubovikova N., Karcher C., Kolesnikov C., 2014, Electromagnetic flow control in liquid metals using Lorentz force techniques, Proceedings in Applied Mathematics and Mechanics, 14, $721-722$

9. England W.G., Emery A.F., 1969, Thermal radiation effects on the laminar free convection boundary layer of an absorbing gas, Journal of Heat Transfer, 91, 1, 37-44, DOI: 10.1115/1.3580116

10. Ganesh Kumar K., Haq R., Rudraswamy N.G., Gireesha B.J., 2017, Effects of mass transfer on MHD three dimensional flow of a Prandtl liquid over a flat plate in the presence of chemical reaction, Results in Physics, 7, 3465-3471

11. Gebhart B., Pera L., 1971, The nature of vertical natural convection flows resulting from the combined buoyancy effects of thermal and mass diffusion, International Journal of Heat and Mass Transfer, 14, 2025-2050

12. Hari R.K., PAtel H.R., 2016, Radiation and chemical reaction effects on MHD Casson fluid flow past an oscillating vertical plate embedded in porous medium, Alexandria Engineering Journal, $\mathbf{5 5}, 583-595$

13. Hayat T., Abbas T., Ayub M., Farooq M., Alsaedi A., 2016, Flow of nanofluid due to convectively heated Riga plate with variable thickness, Journal of Molecular Liquids, 222, 854-862

14. Hayat T., Khan M., Imtiaz M., Alsaedi A., 2017a, Squeezing flow past a Riga plate with chemical reaction and convective conditions, Journal of Molecular Liquids, 225, 569-576

15. Hayat T., Khan M., Khan M.I., Alsaedi A., Ayub M., 2017b, Electromagneto squeezing rotational flow of Carbon $(\mathrm{C})$-Water $\left(\mathrm{H}_{2} \mathrm{O}\right)$ kerosene oil nanofluid past a Riga plate: A numerical study, PLOS ONE, 12

16. Hussanan A., Salleh M.Z., Tahar R.M., Khan I., 2014, Unsteady boundary layer flow and heat transfer of a Casson fluid past an oscillating vertical plate with Newtonian heating, PLOS $O N E, 9$

17. Ibrahim S.Y., Mankinde O.D., 2011, Radiation effect on chemically reacting magneto hydrodynamics (MHD) boundary layer flow of heat and mass transfer through a porous vertical flat plate, International Journal of Physical Sciences, 6, 1508-1516

18. Kumar V.R., Raju M.C., Raju G.S.S., Varma S.V.K., 2016, Thermal diffusive free convective radiating flow over an impulsively started vertical porous plate in conducting field, Journal of Physical Mathematics, 7, 1

19. Magyari E., Pantokratoras A., 2011, Aiding and opposing mixed convection flows over the Riga-plate, Communications in Nonlinear science and Numerical Simulation, 16, 3158-3167

20. Makanda G., Shaw S., Sibanda P., 2015, Effects of radiation on MHD free convection of a Casson fluid from a horizontal circular cylinder with partial slip in non-Darcy porous medium with viscous dissipation, Boundary Value Problems, 2015, 75

21. Pantokratoras A., Magyari E., 2009, EMHD free-convection boundary-layer flow from a Riga-plate, Journal of Engineering Mathematics, 64, 303-315

22. Pattnaik J.R., Dash G.C., Singh S., 2017, Radiation and mass transfer effects on MHD flow through porous medium past an exponentially accelerated inclined plate with variable temperature, Ain Shams Engineering Journal, 8, 67-75 
23. Pramanik S., 2014, Casson fluid flow and heat transfer past an exponentially porous stretching surface in presence of thermal radiation, Ain Shams Engineering Journal, 5, 205-212

24. Raju C.S.K., Sandeep N., Sugunamma V., Babu M.J., Reddy J.V.R., 2016, Heat and mass transfer in magnetohydrodynamic Casson fluid over an exponentially permeable stretching surface, Engineering Science and Technology, an International Journal, 19, 45-52, DOI: 10.1016/j.jestch.2016.06.008

25. Ramesh G.K., Prasannakumara B.C., Gireesha B.J., Rashidi M.M., 2016, Casson fluid flow near the stagnation point over a stretching sheet with variable thickness and radiation, Journal of Applied Fluid Mechanics, 9, 1115-1122

26. Soundalgekar V.M., Ganesan P., 1981, Finite difference analysis of transient free convection with mass transfer on an isothermal vertical flat plate, International Journal of Engineering Science, 19, 757-770

27. Ullah I., Bhattacharyya I., Shafie S., Khan I., 2016, Unsteady MHD mixed convection slip flow of Casson fluid over nonlinearly stretching sheet embedded in a porous medium with chemical reaction, thermal radiation, heat generation/absorption and convective boundary conditions, PLOS ONE, DOI: 10.1371/journal.pone.0165348

28. Venkatesan J., Sankar D.S., Hemalatha K., Yatim Y., 2014, Mathematical analysis of Casson fluid model for blood rheology in stenosed narrow arteries, Journal of Applied Mathematics, DOI: $10.1155 / 2013 / 583809$

Manuscript received May 7, 2018; accepted for print May 30, 2019 